\title{
Telemedicine - is the cart being put before the horse?
}

\section{Nigel R Armfield PhD, MSc, GradCertPubHlth Research Fellow1,2 \\ Sisira K Edirippulige
PhD, MSC \\ Senior Lecturer ${ }^{1}$ \\ Natalie Bradford \\ PhD, MPH \\ Research Fellow ${ }^{1,2}$ \\ Anthony C Smith \\ PhD, MEd \\ Associate Professor ${ }^{1,2}$ \\ 1 Centre for Online Health, School of Medicine, University of Queensland, Brisbane, QLD. \\ 2 Queensland Children's Medical Research Institute, Brisbane, QLD. \\ N.R.Armfield@uq.edu.au}

doi: 10.5694/mjal3.11101

T elemedicine, the use of information and communication technology to deliver clinical services at a distance, although perceived as an innovation, has been discussed in peer-reviewed literature for over 40 years. ${ }^{1}$ While many articles describe the successes and failures of telemedicine, the evidence base for its use is weak. The common view is that it can benefit patients and clinicians, extending services into places where none previously existed. Here, we reflect on the shortcomings of telemedicine research and implementation, and suggest ways to strengthen the quality of evidence in relation to telemedicine (Box).

\section{State of the evidence}

MEDLINE contains links to over 17000 records relating to telemedicine, telehealth or telecare. However, few of these records have found their way into critically appraised summaries such as those in the Cochrane Library, Joanna Briggs stitute Clinical Online Network of Evidence for Care and Therapeutics (JBI COnNECT+) and Database of Abstracts of Reviews of Effect (DARE). Several systematic reviews of telemedicine have been published, including a recent synthesis that critically appraised 80 systematic reviews and concluded that convincing evidence of the effectiveness of telemedicine is limited. ${ }^{2}$ In recent discussions on the United Kingdom's Whole Systems Demonstrator, concerns were raised that the current evidence may be overstated and an insufficient basis on which to invest public funds. ${ }^{3,4}$

Systematic reviews of the cost-effectiveness of telemedicine have produced contradictory findings. One recent review found no conclusive evidence of cost-effectiveness of telemedicine and telecare interventions, ${ }^{5}$ but another reported that video-based telemedicine was cost-effective for home care and access to on-call hospital specialists. ${ }^{6}$ However, local telemedicine between primary care services and hospitals was reported to be not cost-effective, and mixed results were found for providing rural care. ${ }^{6}$ In a review of telemedicine for home care, only one study of good methodological quality was found. ${ }^{7}$ This study showed that providing care by video was more costly than providing care by conventional means. ${ }^{8}$ All three reviews reported that most economic studies had methodological problems. ${ }^{5-7}$

While most studies of telemedicine have been conducted from the health service perspective, and some have been conducted from the societal perspective, little is known about the economics from the patient and carer perspectives. In one of the recent reviews, ${ }^{6}$ only one very old study in this area was identified. ${ }^{9}$

\section{Drivers for telemedicine}

One of the drivers for telemedicine is to improve access to clinical services that would otherwise be unavailable,

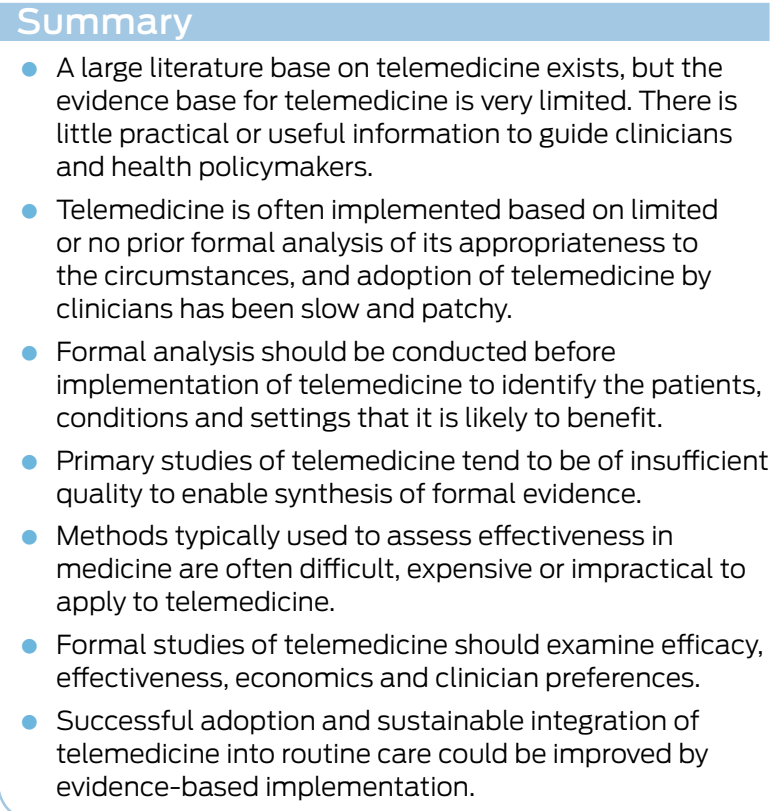

prohibitively expensive, inconvenient or impossible for patients and carers to use. Another is cost reduction for health care providers - for example, from the health system perspective, costs may be reduced when telemedicine avoids the need for expensive patient transport. ${ }^{10}$ However, these savings only occur when the health system bears the cost of transport and the cost of telemedicine. In some cases, while there may be savings for the transport provider and for the referral hospital, the medical and nursing costs at the referring hospital may increase because it must manage the patient who would ordinarily have been transferred out. In these cases, telemedicine may improve care and there may be overall system-level savings, but, from a local budget-holder perspective, telemedicine may actually increase costs. Such economic consequences must be considered when planning a service. While not necessarily considered a driver for telemedicine, inconvenience and out-of-pocket expenses can also be reduced for patients and carers when they avoid a trip to see a practitioner face-to-face. ${ }^{11}$

\section{Adoption and barriers}

Australia has the conditions under which telemedicine should flourish: geography limits access to specialists, technology is readily accessible and inexpensive, and provider reimbursement exists. Yet the uptake of telemedicine has been slow and patchy. ${ }^{12-14}$ While Medicare Benefit Schedule (MBS) item numbers for video-based consultations between general practitioners, nurse practitioners, midwives, Aboriginal health workers and specialists were introduced in July $2011,{ }^{15}$ the uptake of telemedicine outside of the public hospital system has been low. ${ }^{16}$ 
Perhaps this is because practitioners are not convinced of the benefits and there is little incentive to adopt telemedicine, particularly in the absence of clinical and economic evidence. There are also practical problems: scheduling, coordination, integration between public and private health services and interoperability of equipment. The MBS may also require further development: item numbers only cover real-time video interactions, perversely disadvantaging visually oriented specialties such as dermatology, for which store-and-forward telemedicine with still images is generally most appropriate. There is also no reimbursement for the allied health professions. These issues may limit growth of telemedicine in the private sector.

Outside Australia, reimbursement arrangements, medicolegal concerns, and organisational or system barriers also limit the sustainable adoption of telemedicine. ${ }^{17}$

In our experience, one barrier has not been adequately discussed: analysis before implementation of telemedicine is sometimes insufficient or omitted entirely. Unsurprisingly, when telemedicine is implemented to solve a poorly understood problem, or when it is driven by technology rather than the clinical problem, then success, or even the ability to assess success, may be predicated largely on luck. The folly of this approach was noted as long ago as $1995,{ }^{18}$ yet the problem persists.

\section{First things first - understanding the problem}

For telemedicine to improve access and/or control costs, it is essential to understand a priori how it is expected to help; that is, for which patients, which clinical problems and in which settings. It is also important to consider how telemedicine will dovetail with conventional health service delivery, organisation and funding, and fit in with the practice and referral preferences of clinicians. This understanding should go beyond anecdotes; determining whether telemedicine is an appropriate response to a particular set of circumstances should be evidence-based.

As an illustration, while specialists, along with $70 \%$ of the Australian population, choose to live and work in major cities, the remainder of the population is highly distributed. Indeed, relative population growth is greater in regional and remote areas than in major cities. ${ }^{19}$ Patients and carers may travel for several days, and at great expense, to access appropriate care. Also, substantial public funds are spent annually to subsidise patient transport and provide highly specialised emergency retrieval services.

The challenge of geography and population distribution is often referred to as the tyranny of distance. While it seems likely that telemedicine could have a useful role, does this informal notion of "the tyranny" provide sufficient understanding of the problem to justify its implementation? It would be better to analyse health service availability, utilisation and spatial accessibility to determine whether, where and how telemedicine may be useful.

From the economic perspective, because technology costs are decreasing, some services can be provided at little additional cost using software such as Skype and a webcam. However, economic considerations should go beyond the costs of technology. Before funds are consumed on implementation, modelling should be used to predict the changes
Suggestions for developing the evidence base for telemedicine

- First things first - more emphasis should be placed on formally understanding the role, feasibility, efficacy, clinical effectiveness and economics of telemedicine before programs are implemented.

- Evidence-based telemedicine will require a change of mindset in telemedicine research and the techniques that are used to generate evidence. Researchers need to be thinking in the evidence-based medicine paradigm.

- Wherever possible, primary studies should be designed to produce results in a form that may be considered suitable for evidence synthesis.

- Method-comparison studies should be conducted to show the clinical efficacy of telemedicine; formal methods should be used.

- Failures, as well as successes, should be reported in peerreviewed publications.

- More attention should be directed to producing systematic reviews of evidence, evidence summaries and evidencebased recommended practice documents that provide clinicians and health policymakers with practical information. For this to happen, it needs to be recognised that multiple evidence bases exist.

in costs that would occur should telemedicine be implemented. Once a program has been implemented, it is much too late to discover that cost shifting has occurred within, or between, organisations and that the approach has been rendered economically unviable. Published studies of such modelling are rare.

Assuming that it has been determined for which patients and in which settings telemedicine is likely to be helpful, and that it makes economic sense to use telemedicine, it is important to consider the evidence base relating to the efficacy and clinical effectiveness of the proposal. In terms of efficacy - that is, showing that telemedicine produces a beneficial result under ideal conditions - dozens of primary studies have compared diagnostic accuracy or reliability of telemedicine and alternatives under controlled conditions, but few method-comparison studies have formally examined agreement using appropriate methods.

One of most well known and frequently used formal approaches for assessing agreement between two methods of clinical measurements is the Bland-Altman limits of agreement procedure. ${ }^{20} \mathrm{~A}$ search for articles in MEDLINE that mention this approach — using the search terms ["bland-altman" OR "bland altman" OR "limits of agreement"] — returned 8679 results. However, using the search terms [(telemedicine OR telehealth OR telecare OR "remote consultation") AND ("bland-altman" OR "bland altman" OR "limits of agreement")], we found only 30 articles that cite this approach in the telemedicine context. While the Bland-Altman procedure is not appropriate for all comparisons, a rigorous approach to analysis should be taken. Informal assessments of agreement in telemedicine studies are common; many have examined the correlation between telemedicine and traditional approaches. Unsurprisingly, these studies typically show high correlation but say very little about agreement.

If evidence of efficacy is found, the next step is to determine effectiveness - that is, the extent to which telemedicine does what it is intended to do when used in routine 
care. This is particularly challenging because telemedicine is a process, rather than an intervention with clear identifiable outcomes such as a drug or a surgical procedure. It also straddles the disciplines of medicine, information technology and organisational research, which each have different approaches to evaluation. ${ }^{21}$

While randomised controlled trials are considered the gold standard for determining effectiveness in medicine, they are not always appropriate, practical or economical in the telemedicine context. Nor do they necessarily produce information that matches the needs of policymakers. ${ }^{22}$ Heterogeneity between studies typically prevents the pooling of results to analyse effects and hinders the generalisation of findings between settings.

Where formal experimental studies are proposed, they should be preceded by hypothesis-generating pilot studies, but this appears to be uncommon practice.

Finally, before implementation, it is important to pragmatically explore clinicians' opinions on the proportion of work that could be conducted by telemedicine and the types of patients and clinical problems that telemedicine is appropriate for. After all, clinicians are best placed to know who will choose to practice via telemedicine, and a network of advanced technology does not equate to a medical service unless clinicians use it to practice medicine.

\section{Rethinking evidence generation}

When face-to-face consultations are not possible, telemedicine can be used as a substitute (eg, a GP and patient consulting with a specialist). It may also be a convenient adjunct to care (eg, outpatient surgical follow-up where the duration of the consultation may be brief yet travel time is substantial). In some cases, a telemedicine consultation may be superior to a face-to-face consultation. For instance, in mental health, telemedicine may provide safety for the practitioner and be preferred by the patient. In multidisciplinary care, telemedicine may foster teamwork. For example, tertiary and referring hospital clinicians can manage the ongoing rehabilitation of a burns patient as a single team despite distance. It is difficult to envisage an alternative to telemedicine that would enable specialist teams, referring clinicians, patients and carers to interact simultaneously.

With such a range of applications, the outcomes and methodological approaches to evaluation depend on the circumstances, the intention of the consultation (eg, screening, diagnosis, treatment, advice or follow-up) and the acuity of the problem. In some cases, non-inferiority to a faceto-face interaction may be desired. In cases where timely local care is otherwise unavailable, telemedicine may simply need to be better than nothing.

Identifying appropriate methodological approaches to evaluate telemedicine is difficult. In a review of 50 studies concerning methods for assessing telemedicine, it was found that many authors argued the need for larger well designed studies, but few proposed ways to do so. ${ }^{21}$ This is an important finding; however, a fundamental question begs an answer first: what type of evidence is needed, and who needs it, for telemedicine to mature? For this to be answered, our thinking about telemedicine must also mature - governments, health researchers and practitioners all have important roles in informing the future of telemedicine, yet all are influenced by their own perspectives, agendas and information. ${ }^{22}$ It has been suggested:

There is not one evidence-base but several bases. These disparate bodies of knowledge become multiple sets of evidence that inform and influence policy rather than determine it. ${ }^{23}$

This perhaps underlies a fundamental reason why so few data have been synthesised into useful and accessible evidence: while telemedicine is not new, it remains unclear how best to evaluate it to provide useful and relevant information for the various stakeholders.

\section{Conclusion}

We believe that success in telemedicine is defined as its sustainable integration into routine clinical care. Such integration is complex and unlikely to occur by good luck. What is required is a disciplined, multifactorial, formal assessment of where telemedicine may be useful. This assessment should be done diligently and before implementation. It may be necessary to break the cycle of methodologically weak primary studies and to focus attention on better understanding the evidence needs of clinicians and health system policymakers. In considering how best to produce and communicate evidence, the existence of multiple evidence bases must be recognised and formal empirical results should be synthesised with pragmatic experience-based information. We may then finally have the cart and the horse in their correct configuration.

Competing interests: No relevant disclosures.

Provenance: Not commissioned; externally peer reviewed.

1 Fatehi F, Wootton R. Telemedicine, telehealth or e-health? A bibliometric analysis of the trends in the use of these terms. J Telemed Telecare 2012; 18: 460-464.

2 Ekeland AG, Bowes A, Flottorp S. Effectiveness of telemedicine: a systematic review of reviews. Int J Med Inform 2010; 79: 736-771.

3 McCartney M. Show us the evidence for telehealth. BMJ 2012; 344: e469.

4 Oliver D. Over-claiming the evidence for telehealth and telecare [letter]? BMJ 2013; 346: f3377.

5 Mistry H. Systematic review of studies of the cost-effectiveness of telemedicine and telecare. Changes in the economic evidence over twenty years. J Telemed Telecare 2012; 18: 1-6.

6 Wade VA, Karnon J, Elshaug AG, Hiller JE. A systematic review of economic analyses of telehealth services using real time video communication. BMC Health Serv Res 2010; 10: 233.

7 Peeters JM, Mistiaen P, Francke AL. Costs and financial benefits of video communication compared to usual care at home: a systematic review. J Telemed Telecare 2011; 17: 403-411.

8 Moreno L, Dale SB, Chen AY, Magee CA. Costs to Medicare of the Informatics for Diabetes Education and Telemedicine (IDEATel) home telemedicine demonstration: findings from an independent evaluation. Diabetes Care 2009; 32: 1202-1204.

9 Oakley AM, Kerr P, Duffill M, et al. Patient cost-benefits of realtime teledermatology - a comparison of data from Northern Ireland and New Zealand. J Telemed Telecare 2000; 6: 97-101.

10 Armfield NR, Donovan T, Bensink ME, Smith AC. The costs and potential savings of telemedicine for acute care neonatal consultation: preliminary findings. J Telemed Telecare 2012; 18: 429-433.

11 Loh PK, Sabesan S, Allen D, et al. Practical aspects of telehealth: financial considerations. Intern Med J 2013; 43: 829-834.

12 Wade V, Eliott J, Karnon J, Elshaug AG. A qualitative study of sustainability and vulnerability in Australian telehealth services. Stud Health Technol Inform 2010; 161: 190-201.

13 Zanaboni P, Wootton R. Adoption of telemedicine: from pilot stage to routine delivery. BMC Med Inform Decis Mak 2012; 12: 1. 
14 Smith AC, Armfield NR, Croll J, Gray LC. A review of Medicare expenditure in Australia for psychiatric consultations delivered in person and via videoconference. J Telemed Telecare 2012; 18: 169-171.

15 Australian Government Department of Health. MBS video consultation items. http://www.mbsonline.gov.au/internet/mbsonline/publishing.nsf/ Content/connectinghealthservices-itemlist (accessed Nov 2013).

16 Smith AC, Armfield NR, Gray LC. Retrospective review of Medicare reimbursement for telehealth in Australia [abstract]. In: Abstracts from the American Telemedicine Eighteenth Annual International Meeting and Exposition. Telemed JE Health 2013; 19: Al-Al34.

17 Gagnon MP, Duplantie J, Fortin JP, Landry R. Implementing telehealth to support medical practice in rural/remote regions: what are the conditions for success? Implement Sci 2006; 1: 18.
18 Coiera E. Medical informatics. BMJ 1995; 310: 1381-1387.

19 Australian Bureau of Statistics. Regional population growth, Australia, 2012-13. Canberra: ABS, 2013. (ABS Cat. No. 3218.0.) http://www.abs.gov. au/ausstats/abs@.nsf/mf/3218.0 (accessed Dec 2013).

20 Bland JM, Altman DG. Statistical methods for assessing agreement between two methods of clinical measurement. Lancet 1986; 1: 307-310.

21 Ekeland AG, Bowes A, Flottorp S. Methodologies for assessing telemedicine: a systematic review of reviews. Int J Med Inform 2012; 81: 1-11.

22 Nutbeam D, Boxall AM. What influences the transfer of research into health policy and practice? Observations from England and Australia. Public Health 2008; 122: 747-753.

23 Head BW. Three lenses of evidence-based policy. Aust J Publ Admin 2008; 67: 1-11.

\section{- Are you getting InSight?}

\section{MJA InSight}

\section{NEWS AND RESEARCH · COMMENT • INSIGHTS}

MJA InSight is a free, weekly

e-newsletter, from the publishers

of the Medical Journal of Australia,

which allows you to click through

to the user-friendly InSight website

for the latest news and views on

medicine and health.
With access to the MJA's expertise, knowledge and resources. MJA InSight provides you with analysis of clinical news from peer-reviewed journals. informed comments from Australia's leading health and medical experts. and thought-provoking blogs.
Poll

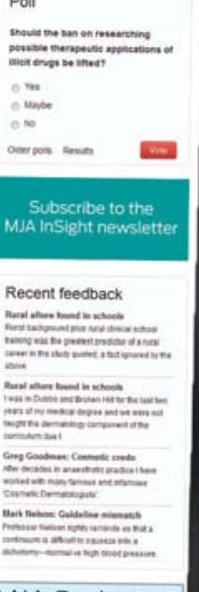

MJAPodcasts

\section{- It is free to register - just go to https://www.mja.com.au/user/register?subscribe=insight}

